\title{
Degeneración cerebelosa subaguda asociada a neoplasia de ovario: presentación de tres casos y revisión de la literatura
}

\author{
J. Remon Masip ${ }^{1}$, A. Gascó Hernández ${ }^{2}$, A. García Arias², R. Guardeño ${ }^{1}$, A. Badia Cantó ${ }^{1}$, \\ T. Cardona Hernández ${ }^{1}$, P. Lianes Barragan ${ }^{1}$
}

\section{Resumen}

Los síndromes neurológicos paraneoplásicos (SNP) son entidades complejas y pueden afectar al $1 \%$ de la población oncológica, con un curso invalidante. La degenerción cerebelosa subaguda es uno de los que se asocia al cáncer de ovario. Presentamos 3 casos clínicos de cáncer de ovario con degeneración cerebelosa subaguda y una breve revisión de la literatura.

Palabras clave: Degeneración cerebelosa subaguda. Cáncer de ovario.

Oncología, 2006; 29 (9):382-386
${ }^{1}$ Servicio de Oncología Médica. Hospital de Mataró. Consorci Sanitari del Maresme. Mataró (Barcelona)

2 Servicio de Oncología Médica. Hospital del Mar. IMAS. Barcelona (España)

\section{Summary}

Neurological paraneoplastic syndromes are complex entities affecting $1 \%$ of oncological patients. Among them, progressive cerebellar degeneration may be associated with ovarian cancer. We present three clinical cases of ovary cancer with progressive cerebellar degeneration, and make a review of the literature.

Key words: Ovary cancer. Progressive cerebellar degeneration. Paraneoplastic syndromes. 


\section{Introducción}

Los síndromes neurológicos paraneoplásicos (SNP) se refieren a aquellas alteraciones neurológicas relacionadas con un cáncer y que no son consecuencia de la invasión directa del tumor, de la diseminación a distancia, de los efectos secundarios del tratamiento quimio o radioterápico ni de las complicaciones cerebro vasculares, infecciosas, metabólicas o nutricionales propias de estos pacientes ${ }^{1}$. Su frecuencia es baja, y sólo afecta a un 1\% de los pacientes $^{2}$. Las neoplasias más frecuentemente asociadas a SNP son: cáncer de pulmón de célula pequeña, mama, ovario, colon, linfoma de Hodgkin y timoma $^{3}$. Los SNP normalmente son severos, invalidantes y en algunos casos letales ${ }^{4}$.

Las alteraciones son producidas probablemente por alteraciones inmunológicas asociadas a la expresión de anticuerpos (Ac) específicos frente antígenos ( $\mathrm{Ag}$ ) ectópicos expresados por el tumor en el del sistema nervioso ${ }^{5}$. El papel de éstos en la génesis de la disfunción neurológica, ha permitido clasificar a los SNP en 3 grupos: a) SNP mediados por anticuerpos que reaccionan frente antígenos onconeuronales de superficie celular (canales iónicos o receptores); b) SNP asociados a Ac frente a Ag onconeuronales, pero probablemente causados por citotoxicidad mediada por linfocitos T; c) SNP mediados por Ac o depósitos de inmunoglobulinas producidos por tumores ${ }^{6}$. Debemos mencionar que para el diagnóstico de este síndrome la presencia de Ac no es imprescindible ni que la positividad de los mismos presuponga su desarrollo.

\section{Casos clínicos}

A continuación presentamos los casos de 3 pacientes afectadas de neoplasia de ovario que presentaron un SNP tipo degeneración cerebelosa subaguda, dos con serología positiva y la otra con serología negativa:

1) Paciente de 61 años sin antecedentes patológicos de interés, que consultó por aparición súbita de ataxia, habla escándida y disfagia de 3 días de evolución. En la exploración física destacaba índice de Karnofsky del $60 \%$, y la presencia de adenopatías laterocervicales izquierdas de $1,5 \mathrm{~cm}$ de tamaño móviles e indoloras. Se procedió a la realización de
TC cerebral que no mostró alteraciones, resonancia magnética (RM) cerebral-orofaríngea inicial con adenopatías en triángulo posterior izquierdo y sin alteraciones a nivel cerebeloso (en la RM realizada posteriormente se apreció atrofia cerebelosa), analítica general sin alteraciones y marcadores tumorales en los que destacaba CA $12.5>6000 \mathrm{UI} / \mathrm{ml}$ y CEA < 0'5 UI/ml. Se realizó TAC tóraco-abdomino-pélvico que mostró adenopatías intra y extraabdominales junto con un elemento nodular heterogéneo de $38 \mathrm{~mm}$ en área anexial derecha El análisis del LCR fue normal con positividad para el Ac anti-Yo tanto en LCR como en plasma. La PAAF de las adenopatías laterocervicales resultó positiva para cistoadenocarcinoma mucinoso de ovario con citoqueratinas 7 y 20 positivas. Con el diagnóstico de cáncer de ovario estadio IV asociado a una degeneración cerebelosa subaguda se procedió a la administración de tratamiento citostático con CBDCA $\left(\mathrm{AUC}=5{ }^{\prime} 5\right)+$ Paclitaxel $\left(175 \mathrm{mg} / \mathrm{m}^{2}\right)$ cada 21 días, apreciándose respuesta biológica y clínica (desaparición de las adenopatías laterocervicales que impidió realización de biopsia) después del primer ciclo. Asimismo no se apreció remisión de su clínica neurológica después de 3 ciclos de tratamiento citostático. La paciente presentó lenta progresión de su clínica neurológica que fue completamente invalidante para las actividades de la vida diaria, con progresión biológica sin signos radiológicos de enfermedad, siendo éxitus a los 10 meses de su diagnóstico.

2) Paciente de 42 años de edad sin antecedentes patológicos de interés diagnosticada en enero de 2002 de neoplasia de ovario estadio IIIC realizándose cirugía no reglada subóptima por lo que se consideró a la paciente tributaria de iniciar tratamiento citostático adyuvante con CBDCA $(\mathrm{AUC}=5,5)+$ Paclitaxel $\left(175 \mathrm{mg} / \mathrm{m}^{2}\right)$ por 7 ciclos, posteriormente se realizó cirugía de intervalo con resección de implantes residuales en omento y cúpula diafragmática. En febrero de 2003 se objetivó progresión biológica asintomática a los 8 meses de finalizar la QT, decidiéndose reiniciar igual esquema citostático por 5 ciclos, seguido de esquema secuencial con Paclitaxel $(100 \mathrm{mg} / \mathrm{m} 2)$ semanal por 6 ciclos. En noviembre del 2003 se evidenció nueva progresión biológica $\left(\mathrm{CA} 12^{\prime} 5=121 \mathrm{UI} / \mathrm{ml}\right)$ con aparición progresiva de inestabilidad cefálica y ataxia. En la exploración física destacaba un IK $80 \%$ junto con las alteraciones neurológicas descritas a las que se añadieron la 
aparición de nistagmus horizonto-rotatorio con la mirada extrema hacia la izquierda, RCP indiferente izquierdo y Romberg positivo bilateral de predominio izquierdo con aumento de la base de sustentación de la marcha. Se realizaron RM cerebral, TAC tóraco-abdominal y estudio del LCR que no mostró alteraciones. Ante la sospecha diagnóstica de SNP se solicitaron anticuerpos anti Yo, Hu, Ri en LCR y plasma que resultaron negativos, pero dado que la clínica era muy sugestiva de degeneración cerebelosa subaguda con serología negativa, la paciente inició tratamiento citostático en esquema con Docetaxel día 1,8 + Gemcitabina día 1, 8 cada 21 días por 7 ciclos, apreciándose una estabilización clínica de su enfermedad oncológica y neurológica con mejoría biológica $(\mathrm{CA} 12 ’ 5=51 \mathrm{UI} / \mathrm{ml})$. En junio 2004 se objetivó nuevamente progresión biológica y neurológica con dificultad para la movilización y disminución de la fuerza en extremidades inferiores, por lo que realizó 5 ciclos de topotecan $2 \mathrm{mg} /$ día, 5 días a la semana con mejoría de la clínica neurológica pero con aumento de los marcadores tumorales. En febrero del 2005, por aparición de astenia grado 3 se decide administrar topotecan semanal, consiguiéndose estabilización de la enfermedad, siendo éxitus en junio de 2005.

3) Paciente de 63 años, con antecedentes de hipertensión, hipercolesterolemia, que en julio de 2003 se diagnosticó de neoplasia de ovario estadio (cistoadenocarcinoma papilar mucinoso) III C con cirugía no reglada y suboptima con un Ca 12.5 post intervención de 345. La paciente realizó 6 ciclos de tratamiento citostático en esquema de Carboplatino $($ AUC $=6)+$ Paclitaxel $\left(175 \mathrm{mg} / \mathrm{m}^{2}\right)$ hasta febrero de 2004 con un Ca 12.5 post tratamiento de $65 \mathrm{UI} / \mathrm{ml}$. En septiembre de 2004 se aprecia progresión biológica con TAC en que se evidenció tumoración de paredes gruesas en la pelvis menor, por lo que se realizó cirugía con resección de la masa con anatomía patológica compatible con el tumor primario. En TAC de control de noviembre de 2004 se evidenciaron adenopatías ilíacas izquierdas y para-aórticas izquierdas junto con nueva progresión biológica $(\mathrm{Ca}$ $12.5=68 \mathrm{UI} / \mathrm{ml})$, desestimándose de nuevo la cirugía por lo que se decidió administrar Tamoxifeno en abril de 2005. En Junio de 2005 la paciente inició marcha atáxica con arreflexia aquílea bilateral y caídas que provocaron fractura humeral derecha, coincidiendo con la retirada del tratamiento hormo- nal por intolerancia digestiva. En agosto de 2005 se realizó RM en la que destacaba atrofia cerebral cortical, atrofia cerebelosa, lesiones isquémicas lacunares localizadas en ambos hemisferios cerebelosos. Asimismo se realizó EMG compatible con neuropatía axonal mixta de predominio sensitivo en extremidades inferiores de carácter moderado. La paciente inició tratamiento con vitamina B12 im y rehabilitación con mejoría parcial de la clínica. En diciembre de 2005 después de cirugía por colecisectomía se apreció empeoramiento de la ataxia por lo que se decidió realizar punción lumbar con citología negativa para células malignas, pero anticuerpos anti-Yo positivos y nueva RM compatible con atrofia cerebelosa con adelgazamiento de las circunvalaciones y agrandamiento del cuarto ventrículo sugestivo de degeneración cerebelosa subaguda. El Ca 12.5 en el momento actual era de $66 \mathrm{mU} / \mathrm{l}$. Dado el intervalo libre de enfermedad se decidió reinducir con igual esquema de tratamiento citostático. La paciente se encuentra en curso de tratamiento con respuesta biológica y estabilización neurológica.

\section{Discusión}

La degeneración cerebelosa subaguda (DCS) se caracteriza por un deterioro cerebeloso simétrico, bilateral, progresivo y de curso subagudo. El principal hallazgo anatomopatológico es una pérdida difusa de células de Purkinje en el cerebelo ${ }^{7}$ sin cambios inflamatorios asociados, así como una degeneración de los núcleos dentado y olivar de los tractos largos de la médula espinal ${ }^{8}$. Los síntomas habitualmente se desarrollan en varias semanas, siendo los más habituales la ataxia de tronco y extremidades, disartria, diplopía y nistagmus. En algunos casos puede existir una alteración del tronco del encéfalo por lo que objetivaremos disfagia y debilidad muscular facial ${ }^{9}$. Este síndrome generalmente precede al diagnóstico ${ }^{10}$ en varios meses. Las pruebas complementarias pueden ser útiles para descartar otras enfermedades de fosa posterior, pero no ofrecen datos distintivos para el diagnóstico de esta entidad. En fases iniciales la RM puede ser normal y posteriormente mostrar atrofia cerebelosa global. El LCR suele mostrar pleocitosis (especialmente en fases iniciales), proteínas aumentadas y secreción intratecal de IgG con bandas oligoclonales que pueden ser 
persistentes a lo largo de la enfermedad ${ }^{11}$. En las pacientes con degeneración cerebelosa las células predominantes en el LCR son células $\mathrm{T}^{12}$ Los Ac antineuronales asociados son: anti-Yo (siendo en un $65 \%$ de los casos, mujeres posmenopáusicas con cáncer de ovario), anti-Tr, anti-Hu y anti-Ri ${ }^{13}$.

El tratamiento consiste en tratar la enfermedad oncológica de base y evitar la respuesta inmune. Cuando el síndrome no mejora a pesar del control del proceso neoplásico, puede ser efectiva la administración de corticoides o la plasmaféresis ${ }^{14}$. Sin embargo, dada la clínica subaguda de la degeneración cerebelosa y el retraso en el diagnóstico y tratamiento, las células de Purkinje destruidas son irrecuperables, por lo que este síndrome raramente mejora con el tratamiento oncológico o la inmunosupresión ${ }^{15}{ }^{16}$.

En este artículo presentamos tres enfermas afectadas de cáncer de ovario con degeneración cerebelosa subaguda asociada. En el primero de ellos, los síntomas preceden al diagnóstico oncológico, tal como ocurre en la mayoría de casos. Esta enferma presentaba positividad para los Ac anti-Yo tanto en plasma como en LCR. Se ha reportado que en los pacientes con serología positiva la evolución del tumor es más indolente y con menor capacidad para desarrollar metástasis que aquellos con serología negativa ${ }^{17}$. En un estudio controlado de la Clínica Mayo con 32 pacientes diagnosticadas de cáncer de ovario estadío III, en las que 8 de ellas presentaban DCS, se objetivó que en este subgrupo de pacientes la carga tumoral al diagnóstico era menor respecto al resto de enfermas, lo que implicaba un mejor pronóstico en cuanto a la respuesta tumoral al tratamiento citostático $(\mathrm{p}=0.05)^{18}$. Esto sugiere que la respuesta inmune contra el tumor retrasa la progresión de la enfermedad de base. La primera enferma presentó tras el primer ciclo de tratamiento citostático una respuesta completa del tumor sin mejoría de la clínica neurológica, tal como suele suceder en las pacientes seropositivas.

Por el contrario, las otras pacientes presentaron durante el seguimiento de su neoplasia de ovario, progresión biológica y aparición subaguda de clínica neurológica compatible con DCS. En el segundo caso no se demostró positividad para los Ac ni en sangre ni en LCR, pero la mejoría clínica y biológica inicial observada apoyaron el diagnóstico de este SNP. A pesar de todo ello, creemos que en el mo- mento actual no disponemos de tratamientos eficaces para estas pacientes.

Así pues, la existencia de una neoplasia indolente y una clínica neurológica abigarrada deben hacernos pensar en un síndrome neurológico paraneoplásico.

Se comunican tres casos de DCS en pacientes afectadas de una neoplasia de ovario que difieren en varios aspectos: cronología del diagnóstico de la DCS, clínica neurológica inicial y positividad de los Ac, siendo la presentación del SNP de la primera paciente la más habitual; ambos casos coinciden en haber presentado una respuesta tumoral objetiva con el tratamiento citostático (más precoz en la paciente con Ac positivos) y una lenta progresión de su clínica neurológica.

\author{
Correspondencia: \\ Dr. J. Remon Masip \\ Servicio de Oncología Médica \\ Hospital de Mataró \\ Carretera de la Cirra, s/n \\ E-08304 Mataró (Barcelona)
}




\section{Bibliografía}

1. Nathanson L, Hall TC. Paraneoplastic Syndromes. Sem Oncol 1997; 24 (3):265-268.

2. Ferrer I. Patología de los síndromes paraneoplásicos del sistema nervioso central, periférico y músculo. Rev Neurol 2000; 31(12):1228-36.

3. Dalmau J, Posner JB. Paraneoplastic Syndromes Affecting the Nervous System. Sem Oncol 1997; 24 (3):318-328.

4. Graus F, Anti-Hu-associated paraneoplastic encephalomyelitis: analysis of 200 patients. Brain 2001;124:1138-48.

5. Carpentier AF, Voltz R, DesChamps T, Posner JB, Dalmau J, Rosenfeld MR. Absence of HuD gene mutations in paraneoplastic small cell lung cancer tissue. Neurology 1998;50:1919.

6. Bataller L, Dalmau J. Anticuerpos antineuronales y síndromes paraneoplásicos. Neurología 2002; 17(2):85-96.

7. Verschuuren J, Chuang L, Rosenblum MK, et al. Inflammatory infiltrates and complete absence of Purkinje cells in anti-Yo associated paraneoplastic cerebellar degeneration. Acta Neuropathol (Berl) 1996; 91:519.

8. Greenlee JE, Brashear HR. Antibodies to cerebellar Purkinje cells in patients with paraneoplastic cerebellar degeneration and ovarian carcinoma. Ann Neurol.1983; 14:609-13.

9. Peterson K, Rosenblum MK, Kotanides H, Posner JB. Paraneoplastic cerebellar degeneration. Neurology 1992; 42:1931-7.

10. Graus F, Keime-Guibert F, Rene R, et al. Anti-Hu associa- ted paraneoplastic encephalomyelitis: analysis of 200 paitents. Brain 2001;124:1138-48.

11. Posner JB. Paraneoplastic syndromes. Neurol Clin 1991; 9:919-36.

12. Albert ML, Austin LM, Darnell RB. Detection and treatment activated $\mathrm{T}$ cells in cerebroespinal fluid of patients with paraneoplastic cerebellar degeneration. Ann Neurol 2000;47:9-17.

13. Chen YT, Rettig WJ, Yenamandra AK, et al. Cerebellar degeneration-related antigen. Proc Natl Acad Sci USA 1990; 87:3077-81.

14. Darnell R, Posner JB, et al. Paraneoplastic syndromes involving the nervous system. N Engl J Med 2003;349:154354.

15. Croteau D, Owainati A, Dalmau J, Rogers LR. Response to cancer therapy in a patient with a paraneoplastic choreiform disorder. Neurology 2001; 57: 719-22,

16. Bataller L, Dalmau J. Paraneoplastic neurologic syndromes:aproches to diagnoses and treatment. Semin. Neurol 2003:23;215-24.

17. Graus F, Dalmau J, Rene R, Tora M, et al. Anti-Hu antibodies in patients with small-cell lung cancer. J Clin Oncol 1997;15:2866-72.

18. Hetzel DJ, Stnahope CR, O’Neill BP, Lennon VA. Gynecologic cancer in patients with subacute cerebellar degeneration predicted by anti-Purkinje's cell antibodies and limited in metastatic volumen. Mayo Clin Proc 1990; 65:1558-1563. 